\title{
Moyamoya Disease Associated with Hyperhomocysteinemia: A Rare Cause of Stroke in Sub-Saharan Africa
}

\author{
Raphael Marie Kabore ${ }^{1 *}$, Habib A. K. Ouiminga², Jean Kabore ${ }^{3}$, Jean-Michel Vallat ${ }^{4}$
}

${ }^{1}$ Department of Neurology, Tengandogo Teaching Hospital, Ouagadougou, Burkina Faso

${ }^{2}$ Department of Neurosurgery, Tengandogo Teaching Hospital, Ouagadougou, Burkina Faso

${ }^{3}$ Department of Neurology, Yalgado Teaching Hospital, Ouagadougou, Burkina Faso

${ }^{4}$ Department of Neurology, Dupuytren Teaching Hospital, Limoges, France

Email: *raphkabore@yahoo.fr

How to cite this paper: Kabore, R.M., Ouiminga, H.A.K., Kabore, J. and Vallat, J.-M. (2022) Moyamoya Disease Associated with Hyperhomocysteinemia: A Rare Cause of Stroke in Sub-Saharan Africa. World Journal of Neuroscience, 12, 22-28.

https://doi.org/10.4236/wjns.2022.121003

Received: May 19, 2021

Accepted: February 6, 2022

Published: February 9, 2022

Copyright $\odot 2022$ by author(s) and Scientific Research Publishing Inc. This work is licensed under the Creative Commons Attribution International License (CC BY 4.0).

http://creativecommons.org/licenses/by/4.0/

\begin{abstract}
Introduction: Moyamoya disease (MMD) is a chronic cerebrovascular disorder characterized by progressive bilateral occlusion of the supraclinoid internal carotid artery (ICA) and its main branches, associated with the development of fine collateral networks, especially adjacent to the site of occlusion in the deep areas of the brain. MMD frequently occurs in East Asian populations, including pediatric and adult patients, and may lead to ischemic or hemorrhagic stroke, headache, epilepsy or transient ischemic attack. The majority is presumed to be of genetic origin and few cases of thrombophilia have been reported in MMD. We report a case of MMD in a young sub-Saharan African woman associated with Hyperhomocysteninemia (HHCys). Observation: A 33-year-old female was seen for aphasia, which had suddenly appeared fourteen days before, and was associated with memory impairment. She mentioned chronic headaches during the 10 previous years, of frontal seat, without migraine criteria. She had no history of hypertension, no obesity, no known dyslipidemia, and there was no family history of stroke. Neurological examination noted Wernicke's aphasia, with discreet right central facial palsy, associated with memory impairment. The brain MRI revealed semi recent infarct in the left parietal lobe, sequelae in the left temporo-occipital and right frontal with lacunar ischemic lesions, associated with several foci of subcortical demyelination. MRA showed stenosis of the supraclinoid carotid arteries and their division branches. In addition, there was a stenosis of the posterior cerebral arteries, with a network of anastomosis at the level of the base of the skull. An investigation of thrombophilia showed hyperhomocysteinemia (HHCys). The diagnosis of moyamoya disease associated with hyperhomocysteinemia was mentioned. She was treated by Aspirin combined to folic acid.
\end{abstract}


After a 3-year setback the patient was asymptomatic. Conclusion: MMD is a rare cause of stroke in sub-Saharan Africa. MRA should be performed whenever possible, as well as investigation for thrombophilia when it's available. Combined antiplatelet agent with folic acid for ischemic stroke prevention is effective.

\section{Keywords}

Moyamoya, Headache, Silent Infarcts, Hyperhomocysteinemia, Diagnosis, Treatment

\section{Introduction}

Moyamoya disease (MMD) is a chronic cerebrovascular disorder characterized by progressive bilateral occlusion of the supraclinoid internal carotid artery (ICA) and its main branches, associated with the development of fine collateral networks, especially adjacent to the site of occlusion in the deep areas of the brain [1]. Moyamoya is a rare disease with reported incidence of 0.086 per 100,000 population [2]. MMD frequently occurs in East Asian populations, including pediatric and adult patients, and may lead to ischemic or hemorrhagic stroke, headache, epilepsy or transient ischemic attack. The prevalence of MMD appears to be slightly lower among Chinese, compared to Koreans or Japanese. According to previous studies, the prevalence of MMD is 10.5/100,000 individuals and the incidence rate is $0.94 / 100,000$ individuals in Japan [3]. There are two peaks of incidence with different clinical presentations, at around 10 years and $30-40$ years [4]. Although a large number of studies have been conducted, the actual etiology of MMD and pathogenesis remain extremely unclear. However, in recent years, ring finger protein $213(R N F 213)$ was identified as a susceptibility gene among East Asian populations [5]. There are few reports describing the classical angiographic appearance of moyamoya vessels with abnormal thrombophilia profile [6]. Homocysteine (HCys) is a non-essential amino acid that derives from the biosynthesis and metabolism of methionine (Met). A high blood level of HCys has been regarded, in the last 10 years, as a biomarker of cardiovascular disease as well as a risk factor for many other pathologies, including Alzheimer's and other dementias [7]. Rare cases of hyperhomocysteinemia (HHCys) associated with MMD have been described [6]. We report a case of moyamoya in a young sub-Saharan Africa woman associated with HHCys.

\section{Observation}

A 33-year-old female was seen for aphasia, which had suddenly appeared fourteen days before, and was associated with memory impairment. She previously reports chronic headaches during the 10 previous years, of frontal seat, without migraine criteria. She had no history of hypertension, no obesity, no known dyslipidemia, and there was no family history of stroke. Neurological examination 
noted Wernicke's aphasia, with discreet right central facial palsy; deep tendon reflexes were normal, there was no sensory disturbance, and cranial nerves were normal. The neuropsychological assessment revealed memory impairment. The brain MRI revealed semi recent infarct on diffusion and FLAIR sequences in the left parietal lobe (Figure 1(A), Figure 1(B)). There were sequelae on left temporal and occipital (Figure 1(C)), and right frontal with lacunar appearance, associated with multiple foci of subcortical demyelination (Figure 1(D)). MRA (Figure 1(E)) showed a stenosis of the intra-cavernous segment of the internal carotids, and posterior cerebral arteries in the P2 segment, most important on the left side, with a network of anastomoses at the base of the skull, achieving a typical "puff of smoke" appearance. Cardiac assessment, ECG, Holter-ECG and cardiac Doppler echocardiography were normal. The thrombophilia work-up showed intermediate hyperhomocysteinemia (HHCys) with a rate of $10.25 \mu \mathrm{mlol} / 1$ (normal value less than $10 \mu \mathrm{mol} / \mathrm{l}$ ), controlled at $17.68 \mu \mathrm{mol} / \mathrm{l}$ six months later. Factor II, factor VII, Protein C and S levels were in normal range. Cholesterol level was also in normal range. The diagnosis of moyamoya associated with HHcys was mentioned. The polymorphism C677 for MTFHF gene mutation research was negative. She was given antiplatelet therapy at the rate of $250 \mathrm{mg}$ of Aspirin per day, combined with folic acid at a dose of $15 \mathrm{mg}$ per day. Paracetamol was prescribed at a rate of one gramme three times a day to treat the headaches. Under treatment we observed a rapid regression of facial palsy, and a gradual regression of aphasia. Successive homocysteine (HCys) checks show a normalization of the level seven months later $(8.36 \mu \mathrm{mol} / \mathrm{l})$. After 3-years follow-up under treatment, the headaches have regressed, and the patient had minor disturbances of memory. The last MRA control showed a better blood flow in Willis polygon (Figure 2).

\section{Discussion}

Moyamoya disease (MMD) is a rare idiopathic progressive vaso-occlusive disease characterized by irreversible condition of main blood vessels to the brain as they enter into the skull. Moyamoya was originally considered to affect predominantly people of Asian heritage, but has now been observed throughout the world [2]. MMD is usually associated with condition like Sickle cell disease specially in pediatric African people. There are two peaks of incidence with different clinical presentations, at around 10 years and 30 - 40 years [4]. The clinical symptoms of MMD include transient ischemic attacks, ischemic stroke, hemorrhagic stroke, epilepsy, headache and cognitive dysfunction. The incidence of each symptom varies depending on the age of the patient [3]. Patients with typical angiographic appearance without any known risk factor were considered MMD, while those with some recognized conditions (neurofibromatosis type 1, Down syndrome, cranial irradiation, and sickle cell disease) are classified as moyamoya syndrome [6]. According to the novel guidelines for moyamoya disease, cerebral angiography is considered essential for the diagnosis, and must 


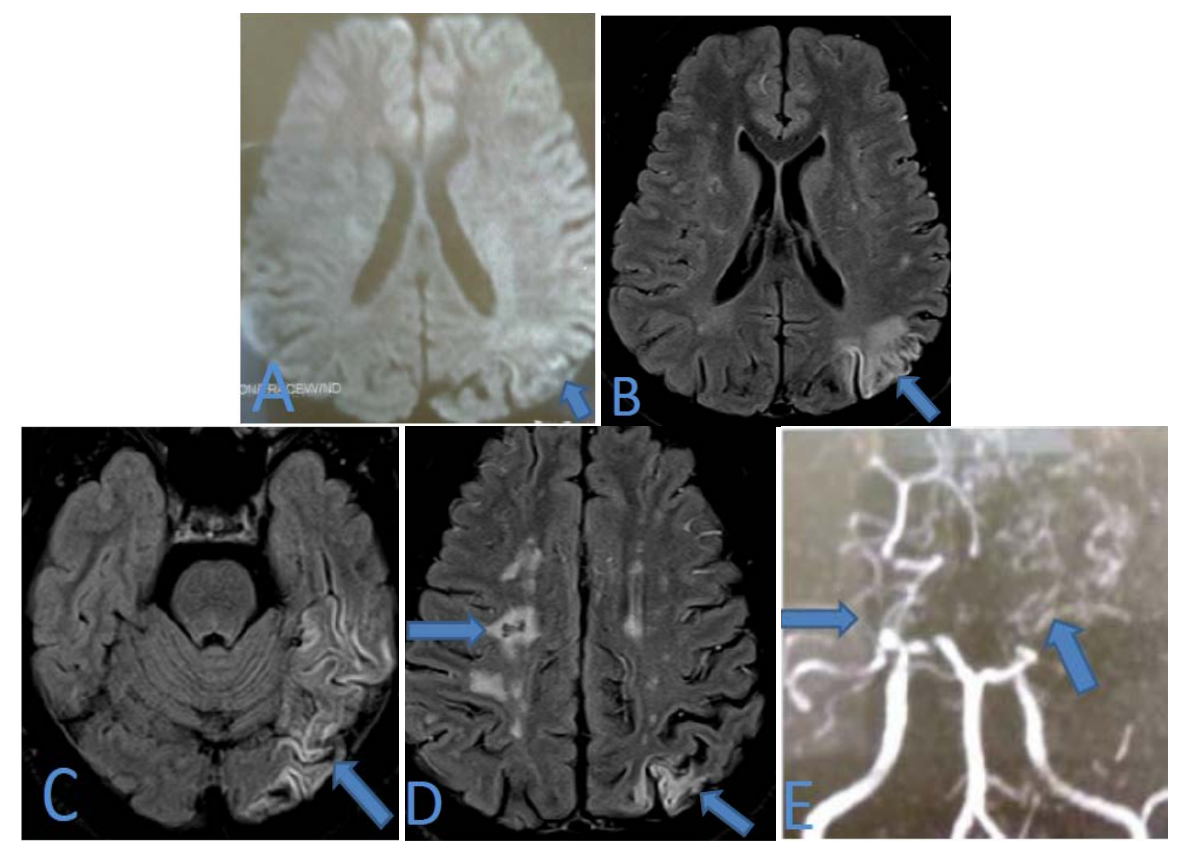

Figure 1. (A) Diffusion sequence showing a moderate hyerintensity (arrow) in the left parietal lobe, evocative of semi recent infarct; (B) The same lesion appear hyperintense (arrow) in the parietal lobe on FLAIR sequence; (C) FLAIR sequence showing sequellar infarction of the left temporal lobe (arrow); (D) Lacunar infarct in the right frontal lobe, associated with multiple foci of subcortical demyelination, (arrows); (E) MRA showing a stenosis of the intra-cavernous segment of right and left internal carotids, and posterior cerebral arteries on P2 segments, most important on the left side, with a network of anastomoses (arrows).

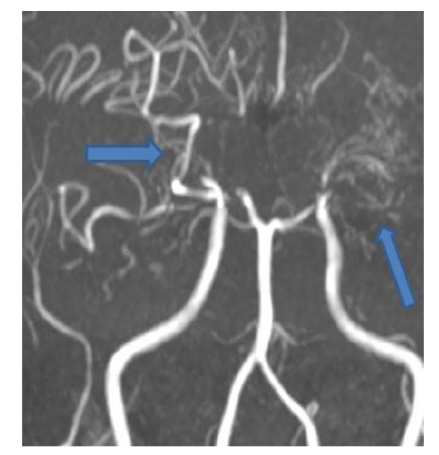

Figure 2. MRA control after 3 years of combined treatment with Asiprin and Folic acid showing a better blood flow in willis polygon than the first exam (Figure $1(\mathrm{E})$ ).

show at least the following findings: 1) Stenosis or occlusion of the terminal portion of the inracranial internal carotid artery or proximal portions of the anterior and/or the middle cerebral artery. 2) Abnormal vascular networks in the vicinity of the occlusive or stenotic lesions in the arterial phase. 3) Bilaterality of findings 1) an 2) [8]. Our patient had a long past of headache without migraine criteria. She also had multiple silent infarcts, which is anusual. The ultimate attack manifested by aphasia and cognitive impairment. Brain MRI revealed the 
semi-recent infarction, coexisting with old demyelinating lesions and infarcts. Angiography revealed a stenosis of the terminal portion of the internal carotids with development of collaterals typical of moyamoya syndrome. Homocysteine (HCys) level was high than normal range. Headaches associated wtih moyamoya disease (HAMD) have previously been reported with features of migraine without aura in a 39-year-old woman [9]. The mechanism of HAMD is uncertain and may be multifactorial. MMD results in narrowing and ultimately, occlusion of intracranial blood vessels. It's possible that HAMD is also caused by this blood vessel pathology. The role of cerebral artery nociceptors in generation of HAMD is supported by studies where stretch of intracerebral vasculature produced nausea, and referred pain in regions typically involved in migraine attacks [9]. Our patient had no criteria of migraine, and suggests the main role of cerebral anoxia linked to cerebral hypoperfusion. Clinically, silent infarcts have not been reported in the literature as we observed in our patient at the time of the diagnosis. This may be due to the localization of lesions in less sensitive areas of the brain. Brain angiography showed carotid terminal portion stenosis, middle cerebral artery, anterior cerebral artery and both posterior cerebral arteries P2 segment, observed at severe stage of the disease. Hyperhomocysteninemia (HHCys) was observed in our patient. The first dosage was intermediate, and the second was high. HHcys associated to MMD has been reported in an 18-year-old woman, who had hemiparesis 8 years ago, and recurrent episodes of vertigo and headaches. She also had seizures during her hospitalization [6]. HHCys is recognized as a risk factor for several diseases, including cardiovascular and neurological conditions. Homocysteine (HCys) is a key metabolite involved in the biosynthesis and metabolism of methionine (Met), which plays a pivotal role in the physiological cell's life cycle. The biochemistry of Met is finely regulated by several enzymes that control HCys concentration. Indeed, balanced activity among the enzymes is essential for the cell's well-being, while its malfunction could raise HCys concentration which can lead to the onset of several pathological conditions [7]. Enzyme defects associated with HCys metabolism are considered the most prevalent cause of HHCys. The enzyme defects has been researched, especially the polymorphisms of the main enzymes involved in HCys metabolism such as Cystathionine b-synthase (CBS) deficiency, Methylenetetrahydrofolate reductase (MTHFR) deficiency, Methionine synthase deficiency, and Methionine adenosyl transferase deficiency [10]. One of the most studied polymorphisms is C677T, present on the gene encoding for the folate-metabolizing enzyme MTHFR. It has been estimated that $10 \%$ of the worldwide population is homozygous (TT genotype) for the common C677T polymorphism, but the frequency can rise up to $25 \%$ in southern Italy and to $32 \%$ in some areas in Mexico. Prolonged exposure to this condition can lead to the onset of cardiovascular disease and can lead to the development of atherosclerosis, stroke, inflammatory syndromes like osteoporosis and rheumatism, as well as neuronal pathologies includin Alzheimer's and Parkinson's diseases [7]. A potential 
mechanism is the thrombotic activity of HCys and its direct effect of endothelial dysfunction, where the HCys acts as an inhibitor of endothelial nitric oxide synthase (eNOS), which will cause reduced bioavailability of NO, through the inhibitory effect of asymmetric dimethylarginine (ADMA) [10]. Observational studies indicated that for each $5-\mu \mathrm{mol} / \mathrm{L}$ rise in serum homocysteine levels, there was a $32 \%$ increased risk of ischemic heart diseases and a 59\% increased risk of stroke. A meta-analysis of randomized controlled trials indicated a $10 \%$ lower risk of stroke and a $4 \%$ lower risk of overall CVD (cardiovascular diseases) with folic acid supplementation [11]. In 2012, the Japenese guidelines recommend using oral antiplatelet agents to medical treatment of MMD. According to them, medical treatment of MMD is roughly classified into treatment of the acute phase of stroke, treatment for preventing recurrence in the chronic phase of stroke, and treatment of asymptomatic moyamoya disease [8]. The most important goal of surgical revascularization is to prevent cerebral infarction by improving cerebral blood flow (CBF) and restoring reserve capacity [12]. However, intra-cranial and extra-cranial revascularization for the prevention of recurrence of bleeding in patients with hemorrhagic MMD is controversial [3]. Our patient received Aspirin $250 \mathrm{mg}$ per day, and folic acid $15 \mathrm{mg}$ per day, resulting in improved cerebral circulation. She no longer had a recurrence of stroke, confirmed by MRA findings. Our report suggests the interest of MRA in the management of stroke in sub-Saharan Africa. MRA remains the reference examination for the diagnosis and follow-up of moyamoya disease. In addition, long-term treatment with antiplatelet agent effectively prevents recurrence of stroke. In the presence of HHCys without polymorphism of MTHFR gene, folic acid is effective to reduce HHCys and prevent recurrence of ischemic stroke and also can improve blood circulation. MMD is underdiagnosed in sub-Saharan Africa due to the unavailability of and inaccessibility of MRI, very often costly for the populations. However, clinicians should use MRI whenever possible so as not to overlook specific rare causes of stroke, especially in young patients. In our practice, Antiplatelet agents should be considered in first intention for MMD ischemic stroke prevention.

\section{Conclusion}

Moyamoya disease (MMD) is a rare cause of stroke in sub-Saharan Africa. We report a case of moyamoya associated with Hyperhomocysteninemia (HHCys) in a sub-Saharan young African woman. MMD is rarely associated with HHCys and most commonly occurs in East Asian populations, with more frequent genetic etiology. Headache may precede cerebral infarctions several years before which can manifest by silent infarction. In the absence of angiography, MRA remains the reference for the study of carotids and their main branches. The study of thrombophilia should be systematically done, especially in young patients. Combined antiplatelet agent with folic acid when HHCys is discovered demonstrates the effectiveness of ischemic stroke prevention. 


\section{Informed Consent}

Written informed consent for publication of clinical details and clinical images was obtained from the patient on Institutional format.

\section{Conflicts of Interest}

The authors declare no conflicts of interest regarding the publication of this paper.

\section{References}

[1] Bersano, A., Guey, S., Bedini, G., Nava, S., Hervé, D., Vajkoczy, P., et al. (2016) Research Progresses in Understanding the Pathophysiology of Moyamoya Disease. Cerebrovascular Diseases, 41, 105-118. https://doi.org/10.1159/000442298

[2] Rohit Kumar, G., Mohtashim, A.Q., Ihtesham, A.Q. and Lakshman, R. (2014) Case Report: A Case Report of Moyamoya Disease in a 36 Year Old African American Woman. F1000Research, 3, 297. https://doi.org/10.12688/f1000research.5859.1

[3] Zhang, H., Zheng, L. and Feng, L. (2019) Epidemiology, Diagnosis and Treatment of Moyamoya Disease (Review). Experimental and Therapeutic Medicine, 17, 1977 1984. https://doi.org/10.3892/etm.2019.7198

[4] Kim, J.S. (2016) Moyamoya Disease: Epidemiology, Clinical Features, and Diagnosis. Journal of Stroke, 18, 2-11. https://doi.org/10.5853/jos.2015.01627

[5] Ma, Y.G., Zhang, Q., Yu, L.B. and Zhao, J.Z. (2016) Role of Ring Finger Protein 213 in Moyamoya Disease. Chinese Medical Journal, 129, 2497-2501. https://doi.org/10.4103/0366-6999.191824

[6] Durga, S.M., Gopal, K.B., Mahadev, M. and Bharat, K.M. (2018) Hyperhomocysteinemia in a Patient with Moyamoya Disease. Case Reports in Neurological Medicine, 2018, Article ID: 7806873. https://doi.org/10.1155/2018/7806873

[7] Tinelli, C., Di Pino, A., Ficulle, E., Marcelli, S. and Feligioni, M. (2019) Hyperhomocysteinemia as a Risk Factor and Potential Nutraceutical Target for Certain Pathologies. Frontiers in Nutrition, 6, 49. https://doi.org/10.3389/fnut.2019.00049

[8] Research Committee on the Pathology and Treatment of Spontaneous Occlusion of the Circle of Willis; Health Labour Sciences Research on Measures for Intractable Diseases (2012) Guidelines for Diagnosis and Treatment of Moyamoya Disease (Spontaneous Occlusion of the Circle of Willis). Neurologia Medico-Chirurgica (Tokyo), 52, 245-266. https://doi.org/10.2176/nmc.52.245

[9] Zach, V., David Bezov, D., Lipton, R.B. and Ashina, S. (2010) Headache Associated with Moyamoya Disease: A Case Story and Literature Review. The Journal of Headache and Pain, 11, 79-82. https://doi.org/10.1007/s10194-009-0181-8

[10] Al Mutairi, F. (2020) Hyperhomocysteinemia: Clinical Insights. Journal of Central Nervous System Disease, 12, 1-8. https://doi.org/10.1177/1179573520962230

[11] Li, Y.P., Huang, T.Y., Zheng, Y., Muka, T., Troup, J. and Hu, F.B. (2016) Folic Acid Supplementation and the Risk of Cardiovascular Diseases: A Meta-Analysis of Randomized Controlled Trials. Journal of the American Heart Association, 5, e003768. https://doi.org/10.1161/JAHA.116.003768

[12] Kim, T., Wan Oh, C., Seung Bang, J., Eun Kim, J. and Cho, W-S. (2016) Moyamoya Disease: Treatment and Outcomes. Journal of Stroke, 18, 21-30. https://doi.org/10.5853/jos.2015.01739 